\title{
An ATP-Binding Cassette Pleiotropic Drug Transporter Protein Is Required for Xenobiotic Tolerance and Antagonism in the Fungal Biocontrol Agent Clonostachys rosea
}

\author{
Mukesh K. Dubey, Dan Funck Jensen, and Magnus Karlsson \\ Uppsala BioCenter, Department of Forest Mycology and Plant Pathology, Swedish University of Agricultural Sciences, \\ Box 7026, 75007 Uppsala, Sweden
}

Submitted 9 December 2013. Accepted 5 March 2014.

\begin{abstract}
ATP-binding cassette (ABC) transporters mediate active efflux of natural and synthetic toxicants and are considered to be important for drug tolerance in microorganisms. In biological control agents (BCA), ABC transporters can play important roles in antagonism by providing protection against toxins derived from the fungal prey and by mediating the secretion of endogenous toxins. In the present study, we generated deletion and complementation strains of the ABC transporter abcG5 in the fungal BCA Clonostachys rosea to study its role in xenobiotic tolerance and antagonism. Gene expression analysis shows induced expression of $a b c G 5$ in the presence of the Fusarium mycotoxin zearalenone (ZEA), secreted metabolites of $F$. graminearum, and different classes of fungicides. Phenotypic analysis of $a b c G 5$ deletion and complementation strains showed that the deletion strains were more sensitive towards $F$. graminearum culture filtrates, ZEA, and iprodione- and mefenoxam-based fungicides, thus suggesting the involvement of abcG5 in cell protection. The $\Delta a b c G 5$ strains displayed reduced antagonism towards $F$. graminearum in a plate confrontation assay. Furthermore, the $\triangle a b c G 5$ strains failed to protect barley seedlings from $F$. graminearium foot rot disease. These data show that the abcG5 ABC transporter is important for xenobiotic tolerance and biocontrol traits in C. rosea.
\end{abstract}

Filamentous fungi have evolved a diverse arsenal of mechanisms to cope with endogenous or exogenous toxic compounds, and one such mechanism is the removal of these compounds by active efflux mediated by ATP-binding cassette (ABC) transporter proteins (Del Sorbo et al. 2000; de Waard et al. 2006). $\mathrm{ABC}$ transporters are present in both prokaryotes and eukaryotes and utilize energy derived from ATP hydrolysis to shuttle metabolites and toxicants across cellular membranes (Del Sorbo et al. 2000; Sharom 2008). The function of ABC transporters depend on four conserved core domains: two cytoplasmic nucleotide binding domains (NBD) and two membrane-associ-

Corresponding author: Mukesh K. Dubey; E-mail: mukesh.dubey@ slu.se; Telephone: +4618 672540; Fax: +4618 673599.

* The $\boldsymbol{e}$-Xtra logo stands for "electronic extra" and indicates that two supplementary figures and one supplementary table are published online and that Figure 3 appears in color online.

(C) 2014 The American Phytopathological Society ated transmembrane domains (TMD). The exception to this rule is the half-size transporters that possess only one NBD and one TMD domain (Biemans-Oldehinkel et al. 2006; Coleman and Mylonakis 2009; Lamping et al. 2010). A single NBD generally contains seven conserved functional motifs that are referred to as the Walker A motif or P-loop, Walker B motif, ABC signature motif or C-loop, Q-loop, pro-loop, Hloop, and D-loop. The TMD (TMD1 and TMD2) consists of 12 transmembrane segments (TMS1 to TMS12) in total, with six transmembrane segments in each TMD (Lamping et al. 2010). On the basis of domain topology and phylogeny, the fungal $A B C$ transporter superfamily is divided into 10 families: ABC-A, ABC-B, ABC-C, ABC-D, ABC-E, ABC-F, ABC-G, ABC-H, Caf16p-like, and YDR061W-like (Kovalchuk and Driessen 2010). Three families (ABC-B, ABC-C, and ABC-G) are shown to be involved in efflux of toxic compounds and are referred to as multidrug resistance protein (MDR), multidrug resistance-associated protein (MRP), and the pleiotropic drug resistance protein (PDR) families, respectively (Coleman and Mylonakis 2009; Kovalchuk and Driessen 2010).

Functional characterizations of ABC transporters in filamentous fungi have shown involvement in cellular detoxification, xenobiotics and heavy metal tolerance, and in pathogenesis (Del Sorbo et al. 2000; de Waard et al. 2006; Coleman and Mylonakis 2009; Lamping et al. 2010). For example, in human pathogens such as Candida spp., Aspergillus fumigatus, and Cryptococcus neoformans, a role of $\mathrm{ABC}$ transporters in resistance to antifungal compounds and in virulence has been demonstrated (de Waard et al. 2006; Coleman and Mylonakis 2009; Lamping et al. 2010; Paul et al. 2013). Similarly, in plant-pathogenic fungi, ABC transporters mediate resistance to fungicides and antimicrobial compounds produced by plants and are involved in virulence (de Waard et al. 2006; Coleman and Mylonakis 2009). Functional analysis of ABC transporters in the rice blast fungus Magnaporthe oryzae demonstrate the role of the $\mathrm{ABC} 1, \mathrm{ABC} 3$, and $\mathrm{ABC} 4$ proteins in tolerance to xenobiotics, cytotoxicity, and oxidative stress and during pathogenesis (Gupta and Chattoo 2008; Sun et al. 2006; Urban et al. 1999). Furthermore, mutants deficient in the ABC transporter gene $G p A B C l$ in the potato tuber pathogen Fusarium sambucinum, Mgatr4 in the wheat leaf blotch pathogen Mycosphaerella graminicola, and Bcatrb, BcatrD, and BcatrA in the necrotrophic fungus Botrytis cinerea displayed reduced tolerance to phytoalexins and fungicides and reduced virulence (de Waard et al. 2006; Coleman and Mylonakis 2009; Del Sorbo et al. 2000; Schouten et al. 2008). 
The fungus Clonostachys rosea is a ubiquitous soilborne ascomycete known for its mycoparasitic, entomopathogenic, and nematophagous abilities ( $\mathrm{Li}$ et al. 2004; Luongo et al. 2005; Rodriguez et al. 2011; Toledo et al. 2006; Zhang et al. 2008). The $C$. rosea IK726 strain, isolated from barley roots infected with $F$. culmorum in Denmark, has proven to be highly efficient as a biocontrol agent (BCA) against fungal pathogens of agricultural and horticultural crops (Jensen et al. 2004, 2007; Knudsen et al. 1995). In order to perform efficiently, BCA fungi must tolerate the cytotoxic and antifungal compounds produced not only by the fungal prey during direct antagonistic interactions but, also, during plant root colonization. However, limited information is available concerning the role of $\mathrm{ABC}$ transporters in $\mathrm{BCA}$ fungi during their interac-
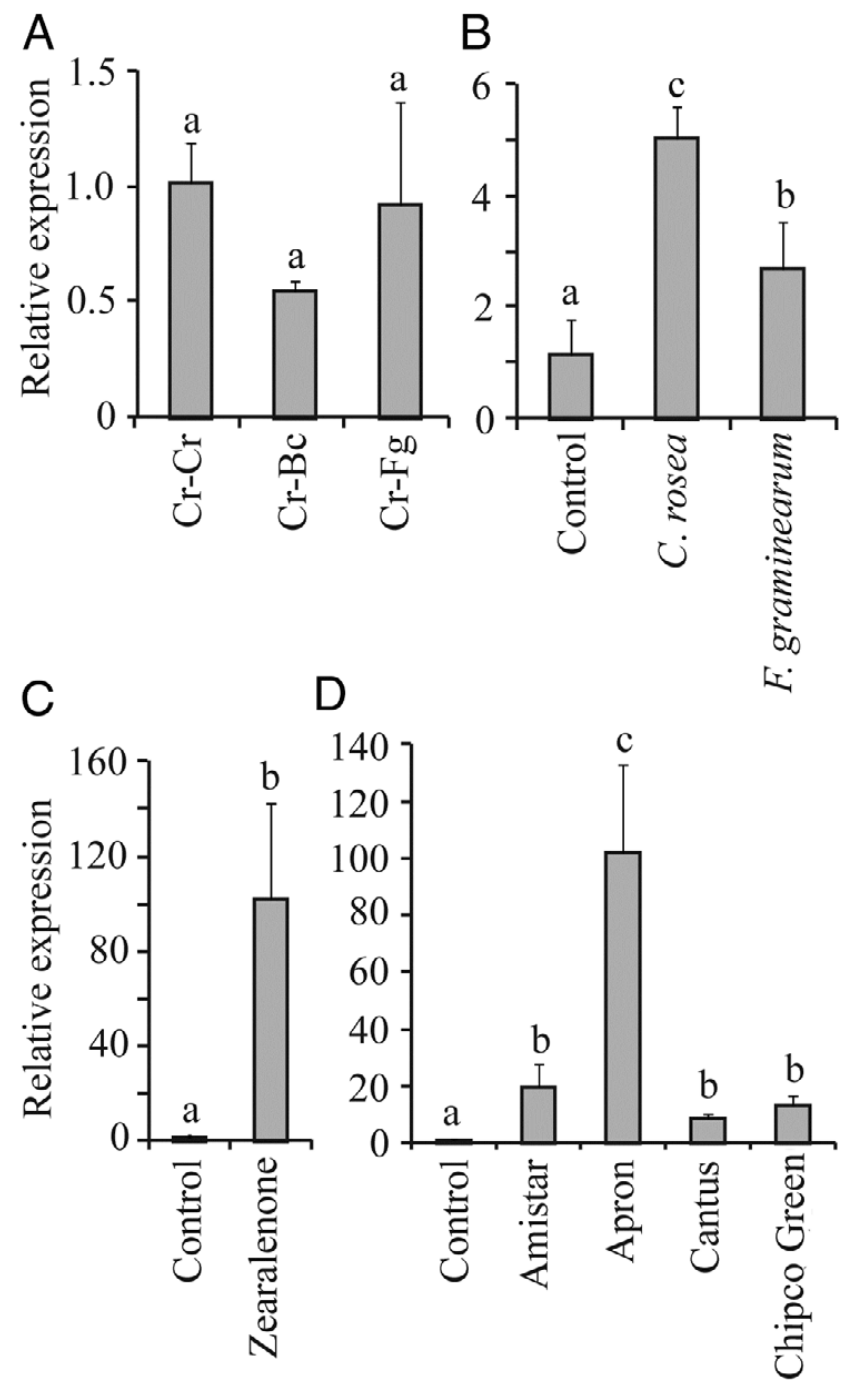

Fig. 1. Expression analyses of abcG5 in Clonostachys rosea. A, During antagonistic interaction and $\mathbf{B}$, in self or $F$. graminearum culture filtrates, mycelia grown in liquid Czapek-Dox (CZ) was used as control. $\mathbf{C}$, In liquid $\mathrm{CZ}$ medium amended with zearalenone (ZEA), $\mathrm{CZ}$ medium amended with methanol was used as control. D, In liquid $\mathrm{CZ}$ medium amended with fungicides, $\mathrm{CZ}$ medium amended with water was used as control. For gene expression analysis during antagonistic interaction, mycelia were harvested at contact stage. For analysis in submerged shake flask cultures, total RNA was extracted from mycelia $2 \mathrm{~h}$ posttreatment in culture filtrates, ZEA, or fungicides. Relative expression levels for $a b c G 5$ in relation to $\beta$-tubulin were calculated from the cycle threshold values and the primer amplification efficiencies, using the formula described by Pfaffl (Pfaffl 2001). Error bars represent standard deviation based on five biological replicates. Different letters indicate statistically significant differences $(P \leq$ 0.05) within experiments based on the Tukey-Kramer test. tions with the fungal prey, other competing microorganisms, and host plants. Identification and functional characterization of the PDR transporter Taabc2 in the mycoparasitic fungus Trichoderma atroviride shows that this PDR transporter is involved in tolerance to fungicides, pathogen toxins, and in antagonism (Ruocco et al. 2009). Recently, deletion of genes encoding $\mathrm{ABC}$ transporters in the entomopathogenic fungus Beauveria bassiana reveals the role of $\mathrm{ABC}$ transporters in oxidative stress tolerance and virulence (Song et al. 2013).

In a recent study, we showed that several $\mathrm{ABC}$ transporter genes that belong to the ABC-B, ABC-C, and ABC-G families are induced in $C$. rosea during exposure to the mycotoxins deoxynivalenol and zearalenone (ZEA) or the fungicide boscalid (Kosawang 2013; Kosawang et al. 2014). In this study, we characterize the biological function of the PDR transporter abcG5 in C. rosea. By analyzing the expression patterns and generating deletion and complementation strains of $a b c G 5$, we demonstrate the role of abcG5 in tolerance to secreted metabolites of $F$. graminearum, the mycotoxin ZEA, and the chemical fungicides Chipco Green and Apron. In addition, our in vitro and in vivo results show that abcG5 is necessary for the antagonistic and biocontrol activity of $C$. rosea against the fungal pathogen $F$. graminearum.

\section{RESULTS}

\section{Sequence analysis of abcG5.}

The full-length sequence of $a b c G 5$ was identified previously (Kosawang et al. 2014). The $a b c G 5$ open reading frame was predicted to encode a polypeptide composed of 1,436 amino acid (aa) residues with a predicted molecular mass of $161 \mathrm{kDa}$ and an estimated isoelectric point at $\mathrm{pH}$ 6.89. The conserved domain analysis of the translated aa sequence using the simple modular architecture research tool (SMART) predicted that the abcG5 protein was composed of two homologous halves, each with an NBD (aa positions 128 to 338 and 823 to 1,014) that precedes a TMD (aa positions 464 to 738 and 1,015 to 1,436 ) with six TMS each. This structure was further verified by conserved domain search. No N-terminal secretion signal peptide was found from analyses using SignalP. The abcG5 protein showed highest similarity (74\%) with the functionally characterized ABC-G family transporter nhABC1 (GenBank accession number XP_003048421.1) from F. solani and Gpabc1 (CAC40023.1) of $F$. sambucinum, followed by $64 \%$ similarity with ifT1 (AET72446) from the entomopathogenic fungus Isaria fumosorosea and $\mathrm{ABC1}$ (AAB86640) from Magnaporthe oryzae. A Clustal W alignment of the $C$. rosea abcG5 sequence with characterized $\mathrm{ABC}$ transporters from filamentous fungi (Botrytis cinerea, CAB52402.1; Aspergilllus nidulans, CAA 93141.1; Mycosphaerella graminicola, XP_003852491.1; Candida albicans, CAA54692.1; F. sambucinum, CAC40023.1) identified the presence of conserved ABC signature (RGVSG GERKRV) and Walker B1 (CWDNSTRGLD) motif but a degenerate Walker A1 (GP/RPGA/SGCT/S) motif in the N-terminal NBD (aa position 128 to 338). The C-terminal NBD consisted of conserved ABC signature (GLNVEQRKRLTIG VEL), Walker B2 (DEPTSGLD), and Walker A2 (GVSGAGK TTLL) motifs (aa position 823 to 1,014).

\section{Expression analysis of $a b c G 5$.}

Real time reverse transcription quantitative polymerase chain reaction (RT-qPCR) on reverse transcribed mRNA showed no difference in abcG5 expression between $C$. rosea self-interaction $(\mathrm{Cr}-\mathrm{Cr})$ and during $C$. rosea interaction with the fungal prey Botrytis cinerea $(\mathrm{Cr}-\mathrm{Bc})$ or $F$. graminearum (Cr-Fg) (Fig. 1A). In order to monitor the response to endogenous and exogenous secreted metabolites, $C$. rosea was inocu- 
lated in culture filtrates from itself ( $C$. rosea), or from $F$. graminearum, or in fresh media. Expression of $a b c G 5$ was significantly $(P \leq 0.011)$ upregulated in $C$. rosea mycelium grown both in self-culture filtrates and $F$. graminearum-culture filtrates compared with the control treatment. Interestingly, a significant $(P<0.001)$ induction of $a b c G 5$ expression was detected in mycelium grown in self culture filtrates in relation to F. graminearum culture filtrates (Fig. 1B). Furthermore, expressions of $a b c G 5$ were strongly induced $(P<0.001)$ when $C$. rosea was exposed to $\mathrm{ZEA}$ or the fungicides Amistar, Apron, Cantus, and Chipco Green at the tested concentrations (Fig. 1C and D).

\section{Generation of $a b c G 5$ deletion and complementation strains.}

C. rosea $a b c G 5$ deletion mutants were generated by replacing $a b c G 5$ with the hygromycin (hygB) selection cassette by Agrobacterium tumefaciens-mediated transformation (ATMT). Successful gene replacement in mitotically stable mutants was confirmed by PCR, using primers located within the hygB cassette together with primers located upstream and downstream of the construct (Supplementary Fig. S1A), as described in our previous work (Dubey et al. 2012, 2013a and b). PCR fragments of the expected size were amplified in all selected $\Delta a b c G 5$ strains, while no amplification was observed in wild type (WT). Furthermore, RT-PCR experiments using primers specific to the $a b c G 5$ sequence demonstrated the complete loss of $a b c G 5$ transcript in each mutant.

A $\triangle a b c G 5$ strain was complemented with the WT $a b c G 5$ gene through ATMT. Successful integration of the nourseothricin (nat1) selection cassette in mitotically stable mutants was confirmed by PCR amplification of nat 1 (data not shown). RTPCR from randomly selected nat1-positive $a b c G 5$ strains, using $a b c G 5$-specific primer pairs, demonstrated restored $a b c G 5$ transcription, while no transcripts were detected in the parental deletion strains. Three independent $C$. rosea abcG5 mutant strains $\Delta a b c G 5 \mathrm{~A}, \Delta a b c G 5 \mathrm{~B}$, and $\Delta a b c G 5 \mathrm{C}$, along with the WT and the complemented $\Delta a b c G 5+$ strain were used in all phenotype analysis.

\section{Deletion of $a b c G 5$ results}

in decreased tolerance to xenobiotics.

No differences in growth rate were detected between deletion and WT strains on Czapek-Dox (CZ) medium. However, all deletion strains showed a significant $(P<0.001)$ reduction in growth rate on $\mathrm{CZ}$ medium amended with ZEA, Apron, or Chipco Green but not on CZ medium amended with Amistar or Cantus. The growth of deletion strains was completely abolished by Chipco Green, whereas 45 and $33 \%$ reductions in growth rate were measured on Apron and ZEA medium, respectively (Fig. 2). In addition, we observed that colonies of the deletion strains had fluffier morphology on CZ medium, although the complemented strain $\Delta a b c g 5+$ did not show restoration in altered colony morphology (Supplementary Fig. S2).

\section{Deletion of $a b c G 5$ reduces antagonism towards $F$. graminearum.}

In order to investigate the role of abcG5 during antagonism, an in vitro plate confrontation assay was used in which mutants or WT strains and $F$. graminearum were confronted with each other. After 4 days postinoculation, $F$. graminearum exhibited a significant $(P<0.001) 28 \%$ increase in growth rate during confrontation with $\Delta a b c G 5$ strains in comparison with $C$. rosea WT strain (Fig. 3A and $\mathrm{B}$ ). However, no differences in growth rate between $C$. rosea WT and deletion strains were measured during the same condition. On the basis of this result, we hypothesized that reduced antagonistic ability of the $\Delta a b c G 5$ strains towards $F$. graminearum was related to reduced efflux levels of toxic metabolites by the $C$. rosea deletion strains during the plate confrontation test. The hypothesis was tested by measuring the mycelial biomass production of $F$. graminearum in heat-treated (to inactivate secreted enzymes) culture filtrates of $C$. rosea WT, deletion, and complementation strains. Similarly to the plate confrontation assay, $F$. graminearum had significantly $(P \leq 0.016)$ increased $(15$ to $22.5 \%)$ biomass in culture filtrates from $\Delta a b c G 5$ strains in comparison with culture filtrate from the WT strain (Fig. 3C). Furthermore, $C$. rosea strains and $F$. graminearum were allowed to interact with each other for 10 days in dual culture plate confrontation tests. The interaction between WT and $F$. graminearum resulted in an apparent inhibition zone, whereas no such zone appeared during interaction between deletion strains and $F$. graminearum (Fig. 3D). In another set of experiments, mycelial biomass production of $C$. rosea strains was measured in the culture filtrate of $F$. graminearum in order to test the tolerance of $\Delta a b c G 5$ and WT strains to the secreted metabolites from $F$. graminearum. In comparison with the WT, deletion strains had significantly $(P<0.001)$ less $(42 \%)$ biomass in $F$. graminearum culture filtrates (Fig. 3E).

\section{Deletion of $a b c G 5$ reduces biocontrol efficiency against $F$. graminearum.}

The biocontrol effect of WT and mutants strains was measured for $F$. graminearum foot rot disease on barley plants using an in vivo sand seedling test. Our results showed a significant $(P \leq 0.036) 20 \%$ increase of disease severity in barley

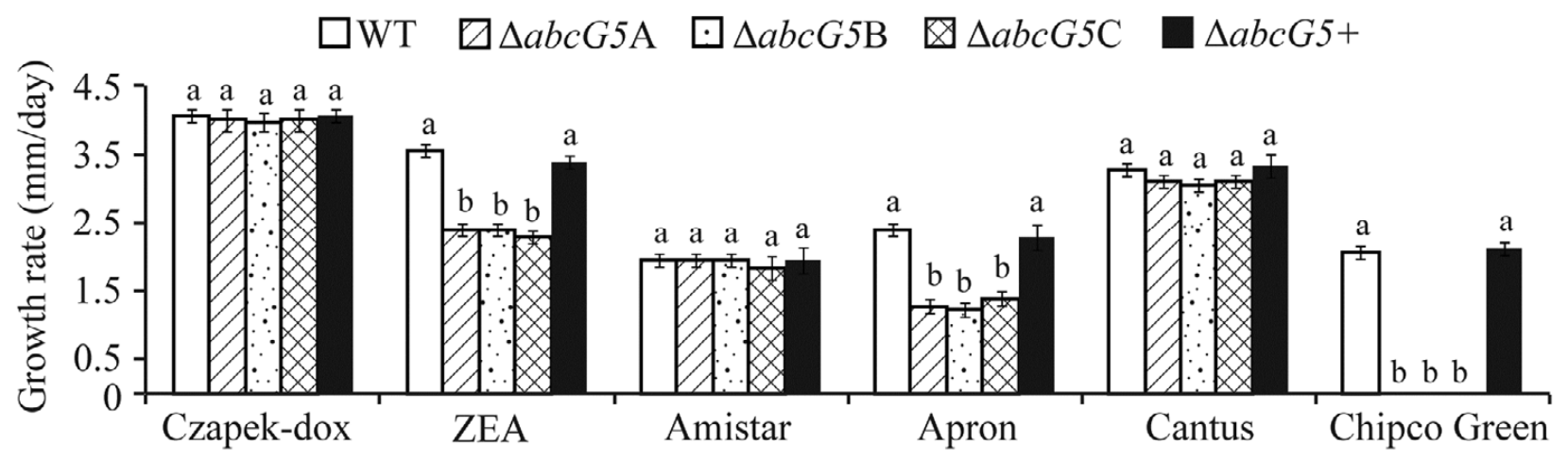

Fig. 2. Growth rate of Clonostachys rosea wild type (WT), deletion, and complemented strains on Czapek-Dox (CZ) medium and CZ medium amended with toxin and fungicides. Strains were inoculated on solid agar medium and were incubated at $25^{\circ} \mathrm{C}$, and the growth rate was recorded 3 days postinoculation. The experiments were carried out in three biological replicates. Different letters indicate statistically significant differences $(P \leq 0.05)$ within experiments based on the Tukey-Kramer test. 
A
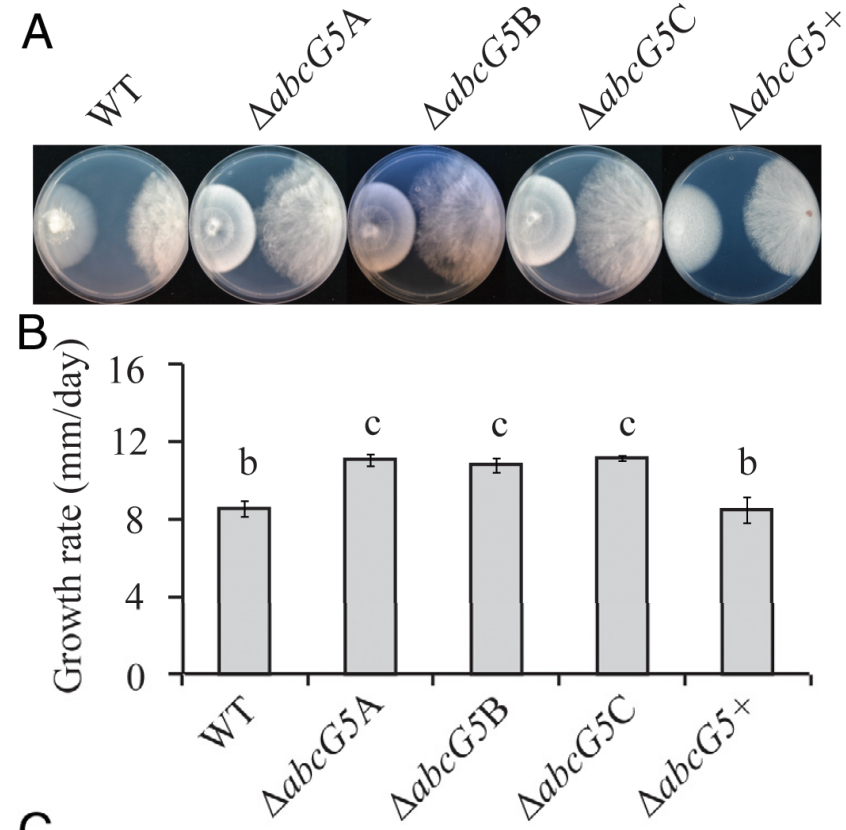

C
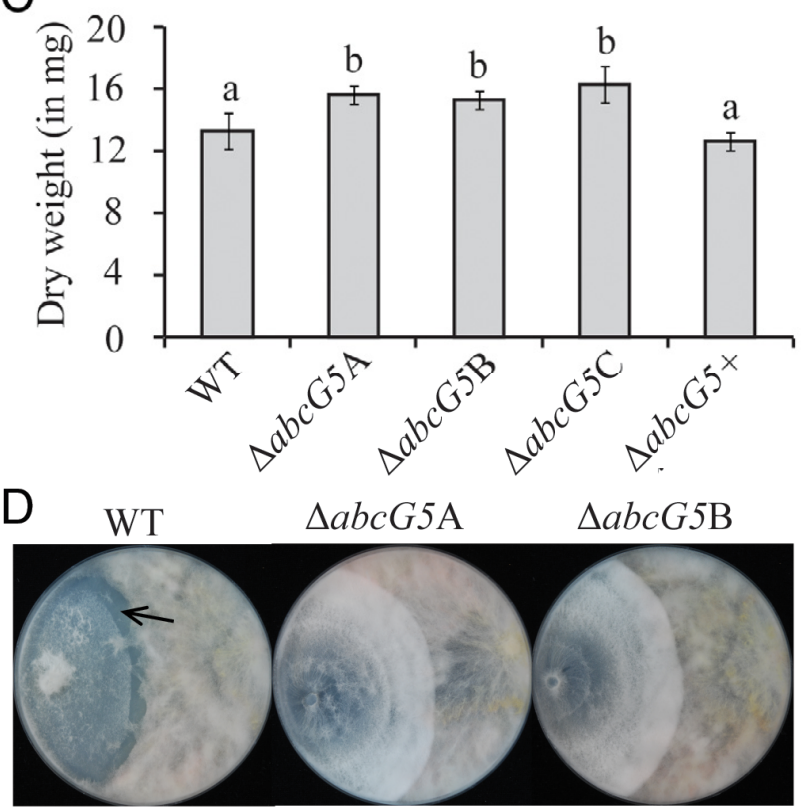

$\triangle a b c G 5 \mathrm{C}$

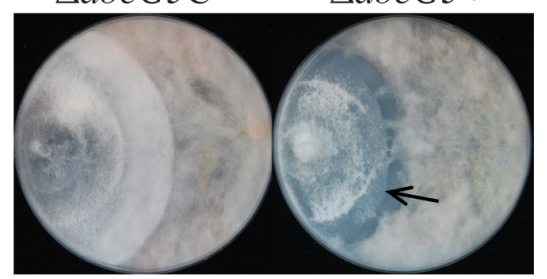

$\mathrm{E}$

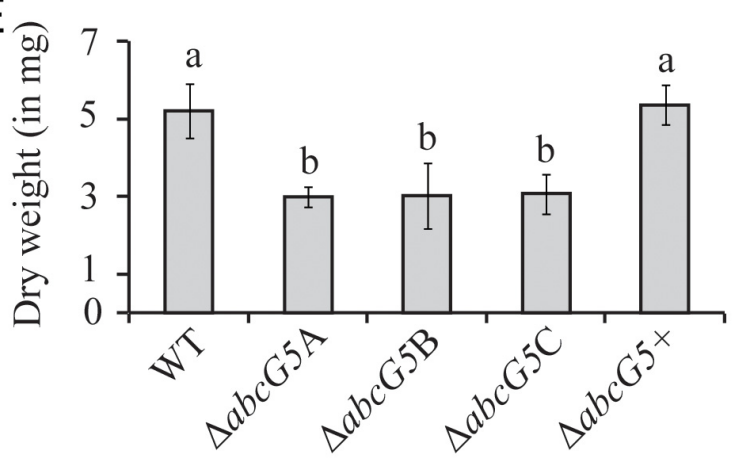

seedlings previously seed coated with $\Delta a b c G 5$ strains as compared with seedlings from seeds coated with WT $C$. rosea. Disease symptoms on seedlings from seeds coated with $\Delta a b c G 5$ strains were, in turn, significantly milder compared with the symptoms on seedlings from control seeds $(F$. graminearum inoculation without any $C$. rosea strain) (Fig. 4).

\section{DISCUSSION}

Recently, $a b c G 5$ was identified in C. rosea, through a suppression subtractive hybridization based transcriptome approach (Kosawang et al. 2014), as being induced by the Fusarium spp. mycotoxin ZEA. In this study, we characterize the physiological functions of abcG5 by generating deletion mutants in $C$. rosea. The $\left(\mathrm{NBD}-\mathrm{TMS}_{6}\right)_{2}$ domain topology of abcG5 is typical for the PDR ABC-G family, which is in line with results from a phylogenetic analysis that placed abcG5 in the ABC-G family (Kosawang et al. 2014). Furthermore, abcG5 show high aa sequence similarity with characterized $\mathrm{ABC}-\mathrm{G}$ family transporters, including Gpabc1 from $F$. sambucinum, nhABC1 from F. solani, ifT1 from Isaria fumosorosea, and $\mathrm{ABC} 1$ from Magnaporthe oryzae. Based on this, it is likely that $a b c G 5$ encodes a $\mathrm{G}$ family PDR $\mathrm{ABC}$ transporter in $C$. rosea.

The mentioned $\mathrm{ABC}$ transporters with high similarity to abcG5 are all involved in tolerance toward various toxic substances. Gpabc1 is required for $F$. sambucinum tolerance to the potato phytoalexin rishitin and, consequently, for virulence on potato tuber (Fleissner et al. 2002). nhABC1, the ortholog to Gpabc1 in F. solani, is involved in tolerance to rishitin and the pea phytoalexin pisatin and acts as a virulence factor on garden pea (Coleman et al. 2011). Furthermore, overexpression of ifT1 in Isaria fumosorosea results in increased tolerance to different types of chemical fungicides, including carbendazim, iprodione, and azoxystrobin (Song et al. 2012). Deletion of $\mathrm{ABC} 1$ results in reduced pathogenicity of Magnaporthe oryzae on rice, although unlike the ifTldeletion mutant in Isaria fumosorosea, the Magnaporthe oryzae $\triangle A B C l$ strain does not display reduced drug tolerance (Urban et al. 1999). Based on the high sequence similarity of abcG5 with the mentioned $\mathrm{ABC}$ transporters, we hypothesize that abcG5 may also be involved in drug tolerance in $C$. rosea.

Mycoparasitic Clonostachys spp. and Trichoderma spp. must be able to withstand toxic metabolites produced both by the mycoparasite itself but also by the fungal prey (Druzhinina

\section{$<$}

Fig. 3. Antagonism test against Fusarium graminearum. A and D, Plate confrontation assay against $F$. graminearum. Agar plugs of Clonostachys rosea strains (left side in the plate) and $F$. graminearum (right side in the plate) were inoculated on opposite sides in 9-cm-diameter agar plates and were incubated at $25^{\circ} \mathrm{C}$. The experiment was performed in three replicates and photographs of representative plates were taken 4 and 10 days postinoculation, respectively. An arrowhead indicates the inhibition zone. B, Growth rate of $F$. graminearum in plate confrontation test against $C$. rosea strains. The experiments were carried out in three biological replicates and the growth rate was recorded 4 days postinoculation. C, Secretion assay of C. rosea strains. Fungal strains were grown in Czapek-Dox (CZ) broth for 10 days at $25^{\circ} \mathrm{C}$. C. rosea culture filtrates were collected after removing mycelia mass, were heated at $80^{\circ} \mathrm{C}$ for $60 \mathrm{~min}$ to inactivate proteins, and were inoculated with a $F$. graminearum agar plug. Biomass production in culture filtrates was analyzed by determining mycelial dry weight 3 days postinoculation. E, Tolerance of $C$. rosea strains to secreted metabolites of F. graminearum. F. graminearum was grown in potato dextrose broth for 10 days at $25^{\circ} \mathrm{C}$. Culture filtrates were collected after removing mycelial mass and were inoculated with $C$. rosea strains. Biomass production in culture filtrates was analyzed in triplicates by determining mycelial dry weight 3 days postinoculation. Experiments were performed in three biological replicates. Different letters indicate statistically significant differences $(P \leq 0.05)$ within experiments based on the Tukey-Kramer test. 
et al. 2011). For example, $C$. rosea produces the ZEA lactonohydrolase zhd101 that specifically detoxifies the antifungal Fusarium spp. mycotoxin ZEA (Takashi-Ando et al. 2002). Furthermore, both $C$. rosea and many Trichoderma spp. have the ability to live in soils as saprophytes and as endophytes in plants (Chatterton et al. 2008; Druzhinina et al. 2011; Lübeck et al. 2002). This may also select for mechanisms to withstand toxic metabolites produced by plants and competing soil microbes. Active efflux, driven by ATP hydrolysis, of toxic metabolites by $\mathrm{ABC}$ transporters with broad substrate specificity is considered to be an important aspect of the initial response of microorganisms during exposure to xenobiotics (Kretschmer et al. 2009). Consequently, broad-spectrum ABC transporters evolved to transport naturally occurring xenobiotics may also provide a certain level of tolerance towards man-made chemicals, such as agricultural pesticides.

C. rosea is able to tolerate relatively high concentrations of various chemical fungicides and pesticides in comparison with recommended commercial doses for controlling plant-pathogenic fungi (Jensen et al. 2011; Macedo et al. 2012; Roberti et al. 2006; Tzelepis and Lagopodi 2011). We hypothesize that $\mathrm{ABC}$ transporters may be one important factor that contributes to this ability, as the draft genome of $C$. rosea IK726 contains 85 different $A B C$ transporters genes (Kosawang 2013). This number is substantially higher than in other filamentous fungi sequenced to date, including mycoparasitic Trichoderma spp. (48 to 62 genes). The numbers of MDR ABC-B, MRP ABC-C, and PDR ABC-G genes are particularly high (21, 19, and 28 genes, respectively), which suggests that $\mathrm{ABC}$ transporters mediate tolerance of $C$. rosea to endogenous and exogenous toxic metabolites.

Induced expression of $a b c G 5$ by ZEA and a diverse group of fungicides, including Amistar, Apron, Cantus, and Chipco Green, indicate a role for abcG5 in efflux of xenobiotics in $C$. rosea. This is verified by the reduced growth rate of $\Delta a b c G 5$ strains on media that contain ZEA, Apron, or Chipco Green. Furthermore, reduction in biomass of $\Delta a b c G 5$ strains in $F$. graminearum culture filtrates suggests that abcG5 is not only involved in efflux of fungicides and ZEA but also in efflux of other secondary metabolites secreted by $F$. graminearum. However, reduction in biomass of $\Delta a b c G 5$ strains in culture filtrates might also be associated with presence of ZEA in secreted metabolites by $F$. graminearum. These data suggest that abcG5 has low substrate specificity and is involved in protecting $C$. rosea from xenobiotics. Similar results are reported previously in the mycoparasitic fungus $T$. atroviride and the entomopathogenic fungus Beauveria bassiana (Ruocco et al. 2009; Song et al. 2013). In T. atroviride, the PDR ABC-G transporter $T a a b c 2$ is found to be upregulated by mycotoxins, fungicides, and pathogen-related metabolites, and tolerance of the Taabc2 deletion mutants to the fungicide Diclorum and metabolites produced by different phytopathogenic fungi is severely compromised (Ruocco et al. 2009).

The contradiction between induced expression of $a b c G 5$ by Amistar and Cantus and the lack of observable growth reduction between $\Delta a b c G 5$ and WT strains on Amistar- and Cantuscontaining media may be explained by an overlap in substrate specificity with other $C$. rosea $\mathrm{ABC}$ transporters that may transport azoxystrobin (active ingredient of Amistar) and boscalid (active ingredient of Cantus). Alternatively, C. rosea may compensate for the $a b c G 5$ deletion by other mechanisms, such as increasing the number of mitochondria, as both azoxystrobin and boscalid target proteins involved in respiration while iprodione (active ingredient of Chipco Green) and mefenoxam (active ingredient of Apron) target lipid biosynthesis and RNA synthesis, respectively. This result is in line with data from Beauveria bassiana in which induced expression of
$P d r l$ and $P d r 3$ by fungicides azoxystrobin, carbendazim, and phosphinothricin was found, while deletion mutants of corresponding genes had significantly reduced growth rates on media amended with carbendazim but not with azoxystrobin (Song et al. 2013).

Our data also suggest that abcG5 may function in secretion of endogenous metabolites that inhibit the growth of $F$. graminearum but not $C$. rosea itself. This is evident as $F$. graminearum grows faster during plate confrontations with the $\Delta a b c G 5$ strains compared to confrontations with the WT. At the same time, the $C$. rosea WT and $a b c G 5$ deletion strains have the same growth rate on both solid $\mathrm{CZ}$ medium and during plate confrontations. The increased biomass production of $F$. graminearum in heat-treated culture filtrates from the $\Delta a b c G 5$ strains compared with the WT emphasize that secreted metabolites rather than secreted enzymes is responsible for this effect. Some peptaibol compounds with antifungal activity are identified in C. rosea (Rodriguez et al. 2011), although it is not known if they are heat stable. However, other peptaibols of fungal origin have been shown to be heat stable (Andersson et al. 2009). Induced expression of T. atroviride Taabc 2 under carbon starvation conditions that trigger secondary metabolite production suggest that this $\mathrm{ABC}$ transporter can also function in secretion of endogenous metabolites. This is validated by the complete loss of ability of the Taabc 2 deletion mutant to secrete the antifungal volatile compound 6-pentyl- $\alpha$-pyrone produced by $T$. atroviride during mycoparasitic interactions (Ruocco et al. 2009). Similar results are also available for the rice blast fungus Magnaporthe oryzae in which inactivation of the MDR ABC-B family gene $A B C 3$ causes accumulation of endogenous toxic compounds in appressoria that results in a reduction in barley leaf penetration but in no effect on viability of the fungal mycelia (Sun et al. 2006).

We show that deletion of abcG5 results in impaired ability to antagonize $F$. graminearum, both in dual plate confrontations and during in vivo interactions on barley seeds and roots. The reduced biocontrol ability may be due to a reduced ability to efflux exogenous toxins produced by $F$. graminearum, a reduced ability to secrete endogenous metabolites, or a combination of both. Reduction in virulence and, consequently, in biocontrol effect as a result from deletion of $\mathrm{ABC}$ transporter genes are reported in biocontrol fungi T. atroviride and Beauveria bassiana (Ruocco et al. 2009; Song et al. 2013). In T.

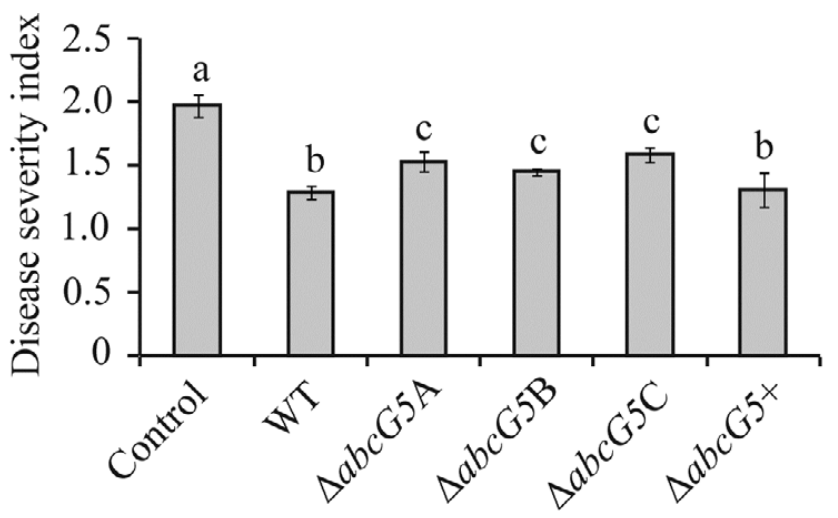

Fig. 4. In vivo bioassay to test the biocontrol ability of Clonostachys rosea strains against Fusarium graminearum foot rot disease on barley, using a sand seedling test. Barley seeds were coated with $C$. rosea conidia and were planted in moist sand together with a $F$. graminearum agar plug. Seedlings were harvested 2 weeks postinoculation, and disease symptoms were scored on a 0 to 4 scale. The experiment was performed in four biological replicates with 10 to 12 plants in each replicate. Different letters indicate statistically significant differences $(P \leq 0.05)$ within experiments based on the Tukey-Kramer test. 
atroviride, deletion of Taabc 2 results in reduced antagonistic ability towards Botrytis cinerea, Rhizoctonia solani, and Pythium ultimum (Ruocco et al. 2009).

It should be noted that the $C$. rosea $\Delta a b c G 5$ strains retain a certain ability to protect barley plants against $F$. graminearum foot rot disease in the current setup, which indicate that additional mechanisms are important for the biocontrol ability of C. rosea. This may include secretion of cell wall-degrading enzymes (Mamarabadi et al. 2008) or toxic peptaibols (Rodriguez et al. 2011). In summary, the data presented in this work demonstrate that abcG5 is a PDR ABC-G transporter that is necessary for xenobiotic tolerance and successful antagonism in C. rosea. It is the first time that an $\mathrm{ABC}$ transporter is shown to be involved in biocontrol in $C$. rosea.

\section{MATERIALS AND METHODS}

\section{Fungal strains and culture conditions.}

C. rosea IK726 (WT) and mutants derived from it and $F$. graminearum 1104-14 were maintained on potato dextrose agar (PDA) medium (Oxoid, Cambridge, U.K.) at $25^{\circ} \mathrm{C} . \mathrm{CZ}$ medium (Sigma-Aldrich, St. Louis) was used to grow fungi for gene expression and phenotypic analysis unless otherwise specified.

\section{Sequence analysis.}

Analyses for conserved domains were performed using the SMART protein analysis tool (Letunic et al. 2009) and conserved domain search (Marchler-Bauer et al. 2011). Signal P version 4.0 was used to search for signal peptide cleavage sites (Petersen et al. 2011). Amino acid sequence alignment was performed using ClustalW2 (Larkin et al. 2007). Analysis for conserved motifs was done manually.

\section{Gene expression analysis.}

Gene expression analysis of $a b c G 5$ was carried out in $C$. rosea under four different conditions: i) during dual culture interaction with $F$. graminearum, ii) in culture filtrates of $C$. rosea and $F$. graminearum, iii) in liquid $\mathrm{CZ}$ medium amended with Fusarium mycotoxin ZEA, and iv) in liquid $\mathrm{CZ}$ medium amended with fungicides Cantus WDG (active ingredient boscalid, anilid group of fungicides; BASF Canada Inc., Mississauga, Canada), Amistar (active ingredient azoxystrobin, methoxyacrylates [strobilurin] group of fungicides; Syngenta, Basel, Switzerland), Chipco Green 75WG (active ingredient iprodione, dicarboximide group of fungicides; Bayer, Leverkusen, Germany), and Apron XL (active ingredient mefenoxam, acylalanins groups of fungicides; Syngenta). ZEA was dissolved in methanol, while fungicides were dissolved in sterile distilled water immediately before use.

For gene-expression analysis during dual culture interactions, the growing front $(7$ to $10 \mathrm{~mm}$ ) of $C$. rosea mycelium was harvested at contact. $C$. rosea confronted with itself was used as control. For gene expression analysis in culture filtrates, an agar plug of $C$. rosea was precultivated for 5 days in 100-ml flasks containing $20 \mathrm{ml}$ of liquid $\mathrm{CZ}$ medium on a rotary shaker (200 rpm [Infors HT Labotron, Rittergasse, Switzerland]) at $25^{\circ} \mathrm{C}$, followed by harvesting by filtration through Miracloth, washing with sterile distilled water, and transfer to new flasks containing $20 \mathrm{ml}$ of culture filtrates of $C$. rosea or $F$. graminearum. Mycelia transferred in fresh $\mathrm{CZ}$ medium were used as control treatment. Culture filtrates were obtained by growing $C$. rosea or $F$. graminearum in $200 \mathrm{ml}$ of liquid $\mathrm{CZ}$ medium in 500-ml flasks as described above. At 10 days postinoculation, biomass was removed by filtering the culture through four layers of Miracloth followed by filter sterilization. For gene-expression analysis in medium containing ZEA or fungicides, culture of $C$. rosea grown for 5 days in $20 \mathrm{ml}$ of liquid $\mathrm{CZ}$ medium was transferred to $\mathrm{CZ}$ media amended with ZEA $(10 \mu \mathrm{g} / \mathrm{ml}$ ), Apron (mefenoxam, $2 \mu \mathrm{g} / \mathrm{ml}$ ), Amistar (azoxystrobin, $7.5 \mu \mathrm{g} / \mathrm{ml}$ ), Chipco Green (iprodione, $250 \mu \mathrm{g} / \mathrm{ml}$ ), or Cantus (boscalid, 2,000 $\mu \mathrm{g} / \mathrm{ml}$ ). In control treatment, ZEA was replaced with an equal volume of methanol and fungicide was replaced by sterile distilled water. After $2 \mathrm{~h}$, fungal mycelia were collected by vacuum filtration, were washed several times in distilled water to remove traces of toxin or fungicides, were frozen in liquid nitrogen, and were stored at $-80^{\circ} \mathrm{C}$.

RNA extraction was done using the Qiagen RNeasy kit following the manufacturer's protocol (Qiagen, Hilden, Germany). After DNaseI (Fermentas, St. Leon-Rot, Germany) treatment, $1 \mu \mathrm{g}$ of total RNA was reverse transcribed in a total volume of $20 \mu \mathrm{l}$, using the Maxima first-stand cDNA synthesis kit (Fermentas). Transcript levels were quantified by RT-qPCR, using the SYBR green PCR master mix (Fermentas) and primer pair abcG5 $\mathrm{F}$ and abcG5 R (Supplementary Table S1) in an iQ5 qPCR system (Bio-Rad, Hercules, CA, U.S.A.), as described previously (Tzelepis et al. 2012). Melt curve analysis was performed after the qPCR reactions, to confirm that the signal was the result from a single product amplification. Relative expression levels for target genes in relation to $\beta$-tubulin, shown previously to be constitutively expressed (Mamarabadi et al. 2008), were calculated from the cycle threshold values and the primer amplification efficiencies by using the formula described by Pfaffl (2001). Gene expression analysis was carried out in five biological replicates, each based on two technical replicates.

\section{Construction of disruption and complementation vectors.}

Genomic DNA was isolated using a hexadecyltrimethylammonium bromide-based method (Nygren et al. 2008). Phusion DNA polymerase (Finnzymes, Vantaa, Finland) was used for PCR amplification of approximately 1-kb $5^{\prime}$ flank and $3^{\prime}$ flank regions of the $a b c G 5$ gene from genomic DNA of $C$. rosea, using primer pairs $A B C$-ups $F$ and $A B C$-ups $R$ and $A B C$-ds $F$ and $A B C$-ds R, respectively. Gateway entry clones of the purified $5^{\prime}$ flank and $3^{\prime}$ flank PCR fragments were generated as described by the manufacturer (Invitrogen, Carlsbad, CA, U.S.A.). The gateway entry clones for hygB $(h p h)$ and natl resistance genes constructed during our previous studies were used (Dubey et al. 2012, 2013a and b). The Gateway LR recombination reaction was performed, using entry plasmid of respective fragments and destination vector pPm43GW (Karimi et al. 2005) to generate the disruption vectors, following the conditions described by the manufacturer (Invitrogen).

An $a b c G 5$ complementation cassette was constructed by PCR amplification of the full-length sequence of $a b c G 5$, including $1-\mathrm{kb}$ upstream and downstream regions from genomic DNA of C. rosea WT, using ABC comp F and ABC comp R. The amplified DNA fragments were purified and integrated into destination vector pPm43GW as described above, using Gateway cloning technology to generate complementation vectors.

\section{ATMT and validation of transformants.}

The disruption and complementation vectors were transformed into Agrobacterium tumefaciens AGL-1 as described before (Dubey et al. 2012). ATMT was performed based on a previous protocol for C. rosea (Utermark and Karlovsky 2008). Transformed strains were selected on plates containing hygB in case of deletion or hygB and nat 1 in the case of complementation. Putative transformants were repeatedly subcultured on PDA plates without the selectable agent five times, followed by re-exposure to selection antibiotics, in order to test for mitotic stability. Mitotically stable colonies were purified by two rounds of single-spore isolation. 
A PCR screening approach of putative transformants was performed to validate the homologous integration of the disruption cassette. The primers used were specific to the $h p h$ gene (P3/P4), sequences flanking the deletion construct ( $\mathrm{ABC}$ ko $\mathrm{F}$ and $\mathrm{ABC}$-ko $\mathrm{R}$ ) and in combination (ABC-ko $\mathrm{F}$ and Hyg $\mathrm{R}, \mathrm{ABC}-\mathrm{ko} \mathrm{R}$ and Hyg F). RT-PCR analysis was conducted on WT, deletion, and complemented strains using RevertAid premium reverse transcriptase (Fermentas) and primer pairs specific for $h p h$ (HygF_qPCR and HygR_qPCR), nat1 (NatF_ qPCR and NatR_qPCR), and $a b c G 5$ (abcG5 F and abcG5 R).

\section{Phenotypic analysis.}

For growth-rate analysis, a 3-mm agar plug from the mycelial front was transferred to solid $\mathrm{CZ}$ medium or $\mathrm{CZ}$ medium containing ZEA $(50 \mu \mathrm{g} / \mathrm{ml}$, dissolved in ethanol) or fungicides with the same concentration of respective active ingredients used for gene expression analysis. Colony diameter was measured after 3 days of growth at $25^{\circ} \mathrm{C}$. Antagonistic behavior against $F$. graminearum was tested using an in vitro plate confrontation assay. An agar plug of $C$. rosea strains were inoculated at the edge of a 9-cm CZ agar plate. After 3 days of incubation at $25^{\circ} \mathrm{C}$, a plug of $F$. graminearum was placed at equal distance to the opposite edge of the plate. F. graminearum growth rate was measured after 4 days of interaction, and photographs of representative plates were taken 10 days postinoculation. For secretion assays, $C$. rosea or $F$. graminearum were grown for 10 days in liquid $\mathrm{CZ}$ or potato dextrose broth on a rotary shaker $(200 \mathrm{rpm})$ at $25^{\circ} \mathrm{C}$, and culture filtrates were obtained as described in the above. Heat inactivation of culture filtrates was performed for $60 \mathrm{~min}$ at $80^{\circ} \mathrm{C}$ (Bae et al. 2011). Heated culture filtrates were cooled to room temperature before inoculation of $F$. graminearum. Agar plugs of $F$. graminearum or $C$. rosea strains were inoculated in 50-ml conical flasks with $20-\mathrm{ml}$ culture filtrates and were incubated at $25^{\circ} \mathrm{C}$ with constant shaking $(100 \mathrm{rpm})$. Biomass production in culture filtrates was analyzed by determining mycelial dry weight 3 days postinoculation. The experiments were performed in three biological replicates unless otherwise specified. The complementation strain $\Delta a b c G 5+$ was included in all phenotype analyses to exclude the possibility that phenotypes derive from ectopic insertions.

\section{Bioassay with $F$. graminearum.}

An in vivo bioassay using a sand seedling test for Fusarium foot rot disease was performed in four biological replicates, and each replicate had 10 to 12 plants for each treatment, following the procedure described previously (Knudsen et al. 1995). In brief, surface-sterilized barley seeds were air dried in a laminar air-flow bench, followed by treatment with $C$. rosea conidia $\left(1 \times 10^{6}\right.$ conidia/ml $)$ in sterile water, for $30 \mathrm{~min}$ on a rotary shaker (100 rpm). Water-soaked seeds were used as control. The seeds were sown in a $1-$ to $2-\mathrm{cm}$ hole, 1 seed in each hole, in moistened sand in individual plastic pots $(5 \times 5 \times 5$ $\mathrm{cm})$. Pathogen inoculation was carried out by placing a $5-\mathrm{mm}$ PDA plug containing $F$. graminearum mycelia close to the seed (Roberti et al. 2008). PDA plugs without $F$. graminearum were used as control. After inoculations, the holes were filled with the moist sand. Pots were kept in a growth chamber with a photoperiod of $12 \mathrm{~h}$ of light, with $150 \mu \mathrm{mol} \mathrm{m} \mathrm{m}^{-1}$ light intensity, and $12 \mathrm{~h}$ of dark, $70 \% \pm 5 \%$ relative humidity, and $15 \pm 1^{\circ} \mathrm{C}$ temperature. Seedlings were harvested 2 weeks postinoculation, and disease symptoms were scored on a 0 to 4 scale described previously (Knudsen et al. 1995): $0=$ healthy plants with no symptoms, $1=$ slightly brown roots and coleoptiles, 2 = moderately brown roots and coleoptiles, $3=$ severely brown roots and coleoptiles, and $4=$ dead plants. The experiment was repeated two times.
Statistical analysis.

Analysis of variance was performed on gene-expression and phenotype data using a general linear model approach implemented in Statistica version 10 (StatSoft, Tulsa, OK, U.S.A.). Pairwise comparisons were made using the Tukey-Kramer method at the $95 \%$ significance level.

\section{ACKNOWLEDGMENTS}

This work was financially supported by the Department of Forest Mycology and Plant Pathology, University of Agricultural Sciences, Agricultural Sciences and Spatial Planning (FORMAS, grant numbers 229-20091530, and 229-2012-1288) and the Danish Agency for Science, Technology and Innovation (DSF grant number 09-063108/DSF) and Carl Trygger Foundation (grant numbers CTS 11:230 and CTS 12:233). The authors thank B. Andersson for providing fungicides.

\section{LITERATURE CITED}

Andersson, M. A., Mikkola, R., Raulio, M., Kredics, L., Maijala, P., and Salkinoja-Salonen, M. S. 2009. Acrebol, a novel toxic peptaibol produced by an Acremonium exuviarum indoor isolate. J. Appl. Microbiol. 106:909-923.

Bae, H., Roberts, D. P., Lim, H.-S., Strem, M. D., Park, S.-C., Ryu, C.-M., Melnick, R. L., and Bailey, B. A. 2011. Endophytic Trichoderma isolates from tropical environments delay disease onset and induce resistance against Phytophthora capsici in hot pepper using multiple mechanisms. Mol. Plant-Microbe Interact. 24:336-351.

Biemans-Oldehinkel, E., Doeven, M. K., and Poolman, B. 2006. ABC transporter architecture and regulatory roles of accessory domains. FEBS (Fed. Eur. Biochem. Soc.) Lett. 580:1023-1035.

Chatterton, S., Jayaraman, J., and Punja, Z. K. 2008. Colonization of cucumber plants by the biocontrol fungus Clonostachys rosea f. catenulata. Biol. Control 46:267-278.

Coleman, J. J., and Mylonakis, E. 2009. Efflux in fungi: La Piece de Resistance. PLoS Pathog. 5:e1000486. Published online.

Coleman, J. J., White, G. J., Rodriguez-Carres, M., and VanEtten, H. D. 2011. An ABC transporter and a cytochrome P450 of Nectria haematococca MPVI are virulence factors on pea and are the major tolerance mechanisms to the phytoalexin pisatin. Mol. Plant-Microbe Interact. 24:368-376.

Del Sorbo, G., Schoonbeek, H., and De Waard, M. A. 2000. Fungal transporters involved in efflux of natural toxic compounds and fungicides. Fungal Genet. Biol. 30:1-15.

de Waard, M. A., Andrade, A. C., Hayashi, K., Schoonbeek, H. J. Stergiopoulos, I., and Zwiers, L. H. 2006. Impact of fungal drug transporters on fungicide sensitivity, multidrug resistance and virulence. Pest Manag. Sci. 62:195-207.

Druzhinina, I. S., Seidl-Seiboth, V., Herrera-Estrella, A., Horwitz, B. A., Kenerley, C. M., Monte, E., Mukherjee, P. K., Zeilinger, S., Grigoriev, I. V., and Kubicek, C. 2011. Trichoderma: The genomics of opportunistic success. Nat. Rev. Microbiol. 9:749-759.

Dubey, M. K., Ubhayasekera, W., Sandgren, M., Jensen, D. F., and Karlsson, M. 2012. Disruption of the Eng $18 B$ ENGase gene in the fungal biocontrol agent Trichoderma atroviride affects growth, conidiation and antagonistic ability. PLoS ONE 7:e36152. Published online.

Dubey, M. K., Broberg, A., Sooriyaarachchi, S., Ubhayasekera, W., Jensen, D. F., and Karlsson, M. 2013a. The glyoxylate cycle is involved in pleotropic phenotypes, antagonism and induction of plant defence responses in the fungal biocontrol agent Trichoderma atroviride. Fungal Genet. Biol. 58-59:33-41.

Dubey, M. K, Broberg, A., Jensen D. F., and Karlsson M. 2013b. Role of the methylcitrate cycle in growth, antagonism and induction of systemic defence responses in the fungal biocontrol agent Trichoderma atroviride. Microbiology 159:2492-2500.

Fleissner, A., Sopalla, C., and Weltring, K.-M. 2002. An ATP-binding cassette multidrug-resistance transporter is necessary for tolerance of $G i b$ berella pulicaris to phytoalexins and virulence on potato tubers. Mol. Plant-Microbe Interact. 15:102-108.

Gupta, A., and Chattoo, B. B. 2008. Functional analysis of a novel ABC transporter ABC4 from Magnaporthe grisea. FEMS (Fed. Eur. Microbiol. Soc.) Microbiol. Lett. 278:22-28.

Jensen, B., Knudsen, I. M. B., Madsen, M., and Jensen, D. F. 2004. Biopriming of infected carrot seed with an antagonist, Clonostachys rosea, selected for control of seedborne Alternaria spp. Phytopathology 94:551-560.

Jensen, D. F., Knudsen, I. M. B., Lübeck, M., Mamarabadi, M., 
Hockenhull, J., and Jensen B. 2007. Development of a biocontrol agent for plant disease control with special emphasis on the near commercial fungal antagonist Clonostachys rosea strain IK726. Aust. Plant Pathol. 36:95-101.

Jensen, B., Knudsen I. M. B., Jensen, D. F., Andersen, B., Nielsen, K. F. Thrane, U., and Larsen, J. 2011. Importance of microbial pest control agents and their metabolites in relation to the natural microbiota on strawberry. Pesticides Research no. 1282011, Danish Environmental Protection Agency, Aarhus, Denmark.

Karimi, M., De Meyer, B., and Hilson, P. 2005. Modular cloning in plant cells. Trends Plant Sci. 10:103-105.

Knudsen, I. M. B., Hockenhul, J., and Jensen, D. F. 1995. Biocontrol of seedling diseases caused by Fusarium culmorum and Bipolaris sorokiniana: Effects of selected fungal antagonists on growth and yield components. Plant Pathol. 44:467-477.

Kosawang, C. 2013. Three-way interaction between Fusarium species, their plant hosts and biocontrol organisms. PhD thesis. Faculty of Science, University of Copenhagen, Denmark.

Kosawang, C., Karlsson, M., Jensen, D. F, Dilokpimol, A., and Collinge, D. B. 2014. Transcriptomic profiling to identify genes involved in Fusarium mycotoxin deoxynivalenol and zearalenone tolerance in the mycoparasitic fungus Clonostachys rosea. BMC Genomics 15:55.

Kovalchuk, A., and Driessen, A. J. M. 2010. Phylogenetic analysis of fungal ABC transporters. BMC Genomics 11:177.

Kretschmer, M., Leroch, M., Mosbach, A., Walker, A. S., Fillinger, S., Mernke, D., Schoonbeek, H., Pradier, J. M., Leroux, P., de Waard, M. A., and Hahn, M. 2009. Fungicide-driven evolution and molecular basis of multidrug resistance in field populations of the grey mould fungus Botrytis cinerea. PLoS Pathog. 5, e1000696. Published online.

Lamping, E., Baret, P. V., Holmes, A. R., Monk, B. C., Goffeau, A., and Cannon, R. D. 2010. Fungal PDR transporters: Phylogeny, topology, motifs and function. Fungal Genet. Biol. 47:127-142.

Larkin, M. A., Blackshields, G., Brown, N. P., Chenna, R., McGettigan, P. A., McWilliam, H., Valentin, F., Wallace, I. M., Wilm, A., Lopez, R., Thompson, J. D., Gibson, T. J., and Higgins, D. G. 2007. Clustal W and Clustal X version 2.0. Bioinformatics 23:2947-2948.

Letunic, I., Doerks, T., and Bork, P. 2009. SMART 6: Recent updates and new developments. Nucleic Acids Res. 37:D229-D232.

Li, G. Q., Huang, H. C., Acharya, S. N., and Erickson R. S. 2004. Biological control of blossom blight of alfalfa caused by Botrytis cinerea under environmentally controlled and field conditions. Plant Dis. 88:12461251.

Lübeck, M., Knudsen, I. M. B., Jensen, B., Thrane, U., Janvier, C., and Jensen, D. F. 2002. GUS and GFP transformation of the biocontrol strain Clonostachys rosea IK726 and the use of these marker genes in ecological studies. Mycol. Res. 106:818-826.

Luongo, L. Galli, M., Corazza, L., Meekes, E. T. M., De Haas, B. H., Plas, L. V., and Köhl, J. 2005. Potential of fungal antagonists for biocontrol of Fusarium spp. in wheat and maize through competition in crop debris. Biocontrol Sci. Techn. 15:229-242.

Macedo, P. E. F., Maffia, L. A., Cota, L. V., Lourenco, V., and Mizubuti, E. S. G. 2012. Sensitivity of four isolates of Clonostachys rosea to pesticides used in the strawberry crop in Brazil. J. Pestic. Sci. 37:333-337.

Mamarabadi, M., Jensen, B., Jensen, D. F., and Lübeck, M. 2008. Realtime RT-PCR expression analysis of chitinase and endoglucanase genes in the three-way interaction between the biocontrol strain Clonostachys rosea IK726, Botrytis cinerea and strawberry. FEMS (Fed. Eur. Microbiol. Soc.) Microbiol. Lett. 285:101-110.

Marchler-Bauer, A., Lu, S., Anderson, J. B., Chitsaz, F., Derbyshire, M K., DeWeese-Scott, C., Fong, J. H., Geer, L. Y., Geer, R. C., Gonzales, N. R., Gwadz, M., Hurwitz, D. I., Jackson, J. D., Ke, Z., Lanczycki, C. J., Lu, F., Marchler, G. H., Mullokandov, M., Omelchenko, M. V., Robertson, C. L., Song, J. S., Thanki, N., Yamashita, R. A., Zhang, D., Zhang, N., Zheng, C., and Bryant, S. H. 2011. CDD: A Conserved Domain Database for the functional annotation of proteins. Nucleic Acids Res. 39:D225-229.

Nygren, C. M. R., Eberhardt, U., Karlsson, M., Parrent, J. L., Lindahl, B. D., and Taylor, A. F. S. 2008. Growth on nitrate and occurrence of nitrate reductase-encoding genes in a phylogenetically diverse range of ectomycorrhizal fungi. New Phytol. 180:875-889.

Paul, S., Diekema D., and Moye-Rowley W. S. 2013. Contributions of Aspergillus fumigatus ATP-binding cassette transporter proteins to drug resistance and virulence. Eukaryot. Cell 12:1619-1628.

Petersen, T. N., Brunak, S., von Heijne, G., and Nielsen, H. 2011. SignalP 4.0: Discriminating signal peptides from transmembrane regions. Nat. Methods 8:785-786.

Pfaffl, M. W. 2001. A new mathematical model for relative quantification in real-time RT-PCR. Nucleic Acids Res. 29:2001-2007.

Roberti, R., Badiali, F., Pisi, A., Pancaldi, D., and Cesari, A. 2006. Sensitivity of Clonostachys rosea and Trichoderma spp. as potential biocontrol agents to pesticides. J. Phytopathol. 154:100-109

Roberti, R., Veronesi, A., Cesaria, A., Casconeb, A., Berardinob, I. D., Bertinib, J., Caruso, C. 2008. Induction of PR proteins and resistance by the biocontrol agent Clonostachys rosea in wheat plants infected with Fusarium culmorum. Plant Sci. 175:339-347.

Rodriguez, M. A., Cabrera, G., Gozzo, F. C., Eberlin, M. N., and Godeas, A. 2011. Clonostachys rosea BAFC3874 as a Sclerotinia sclerotiorum antagonist: Mechanisms involved and potential as a biocontrol agent. J. Appl. Microbiol. 110:1177-1186.

Ruocco, M., Lanzuise, S., Vinale, F., Marra, R., Turra, D., Woo, S. L., and Lorito, M. 2009. Identification of a new biocontrol gene in Trichoderma atroviride: The role of an $\mathrm{ABC}$ transporter membrane pump in the interaction with different plant-pathogenic fungi. Mol. Plant-Microbe Interact. 22:291-301.

Schouten, A., Maksimova, O., Cuesta-Arenas, Y., van den Berg, G., and Raaijmakers, J. M. 2008. Involvement of the ABC transporter BcAtrB and the laccase BcLCC2 in defence of Botrytis cinerea against the broad-spectrum antibiotic 2,4-diacetylphloroglucinol. Environ. Microbiol. 10:1145-1157.

Sharom, F. J. 2008. ABC multidrug transporters: Structure, function and role in chemoresistance. Pharmacogenomics 9:105-127.

Song, T. T., Ying, S. H., and Feng, M. G. 2012. High resistance of Isaria fumosorosea to carbendazim arises from the overexpression of an $\mathrm{ABC}$ transporter (ifT1) rather than tubulin mutation. J. Appl. Microbiol. 112:175-184

Song, T. T., Zhao, J., Ying, S. H., and Feng, M. G. 2013. Differential contributions of five $\mathrm{ABC}$ transporters to mutidrug resistance, antioxidation and virulence of Beauveria bassiana, an entomopathogenic fungus. PLoS ONE 8:62179. Published online.

Sun, C. B., Suresh, A., Deng, Y. Z., and Naqvi, N. I. 2006. A multidrug resistance transporter in Magnaporthe is required for host penetration and for survival during oxidative stress. Plant Cell 18:3686-3705.

Takahashi-Ando, N., Kimura, M., Kakeya, H., Osada, H., and Yamaguchi, I. 2002. A novel lactonohydrolase responsible for the detoxification of zearalenone: Enzyme purification and gene cloning. Biochem. J. 365:1-6

Toledo, A. V., Virla, E., Humber, R. A., Paradell, S. L., and Lastra, C. C. 2006. First record of Clonostachys rosea (Ascomycota: Hypocreales) as an entomopathogenic fungus of Oncometopia tucumana and Sonesimia grossa (Hemiptera: Cicadellidae) in Argentina. J. Invertebr. Pathol. 92:7-10.

Tzelpis, G. D., and Lagopodi, L. A. 2011. Interaction between Clonostachys rosea IK726 and Pseudomonas chlororaphis PCL 1391 against tomato foot and root rot caused by Fusarium oxysporium f. sp. radicis lycopersici. IOBC/wprs Bulletin 63:75-79.

Tzelepis, G. D., Melin, P., Jensen, D. F., Stenlid, J., and Karlsson, M. 2012. Functional analysis of glycoside hydrolase family 18 and 20 genes in Neurospora crassa. Fungal Genet. Biol. 49:717-730.

Urban, M., Bhargava, T., and Hamer, J. E. 1999. An ATP-driven efflux pump is a novel pathogenicity factor in rice blast disease. EMBO (Eur. Mol. Biol. Organ.) J. 18:512-521.

Utermark, J., and Karlovsky, P. 2008. Genetic transformation of filamentous fungi by Agrobacterium tumefaciens. Nat. Protoc. doi:10.1038/ nprot.2008.83. Published online.

Zhang, L., Yang, J., Niu, Q., Zhao, X., Ye, F., Liang, L., and Zhang, K. Q. 2008. Investigation on the infection mechanism of the fungus Clonostachys rosea against nematodes using the green fluorescent protein. Appl. Microbiol. Biotechnol. 78:983-990. 